\title{
Planetary nebulae and their mimics: The MASH-MEN Project
}

\author{
Rozenn Boissay ${ }^{1,2,3}$, Quentin A. Parker ${ }^{2,3,4}$, David J. Frew ${ }^{2,3}$ and \\ Ivan Bojicic ${ }^{2,3}$ \\ ${ }^{1}$ Observatoire Astronomique, Université Louis Pasteur, 67000 Strasbourg, France \\ email: rozenn.boissay@gmail.com \\ ${ }^{2}$ Department of Physics, Macquarie University, North Ryde, NSW 2109, Australia \\ email: quentin . parker@mq. edu . au \\ ${ }^{3}$ Macquarie University Research Centre in Astronomy, Astrophysics \& Astrophotonics \\ ${ }^{4}$ Australian Astronomical Observatory (AAO), Epping, NSW 1710, Australia
}

\begin{abstract}
The total number of true, likely and possible planetary nebulae (PN) now known in the Milky Way is about 3000, approximately twice the number known a decade ago. The new discoveries are a legacy of the recent availability of wide-field, narrowband imaging surveys, primarily in the light of $\mathrm{H} \alpha$. The two most important are the AAO/UKST SuperCOSMOS $\mathrm{H} \alpha$ survey SHS and the Isaac Newton photometric $\mathrm{H} \alpha$ survey IPHAS, which are responsible for most of the new discoveries. A serious problem with previous PN catalogs is that several different kinds of astrophysical objects are able to mimic PN in some of their observed properties leading to significant contamination. These objects include H II regions and Strömgren zones around young $\mathrm{O} / \mathrm{B}$ stars, reflection nebulae, Wolf-Rayet ejecta, supernova remnants, HerbigHaro objects, young stellar objects, B[e] stars, symbiotic stars and outflows, late-type stars, cataclysmic variables, low redshift emission-line galaxies, and even image/detector flaws. PN catalogs such as the Macquarie/AAO/Strasbourg $\mathrm{H} \alpha$ Planetary Nebula catalog (MASH) have been carefully vetted to remove these mimics using the wealth of new wide-field multi-wavelength data and our $100 \%$ follow-up spectroscopy to produce a compilation of new PN discoveries of high purity. During this process significant numbers of PN mimics have been identified. The aim of this project is to compile these MASH rejects into a catalog of Miscellaneous Emission Nebulae (MEN) and to highlight the most unusual and interesting examples. A new global analysis of these MEN objects is underway before publishing the MEN catalog online categorizing objects by type together with their spectra and multi-wavelength images.
\end{abstract}

Keywords. catalogs, planetary nebulae: general, stars: AGB and post-AGB, stars: fundamental parameters, surveys, techniques: photometric, techniques: spectroscopic, methods: data analysis

\section{Introduction}

The MASH Miscellaneous Emission Nebulae catalog (MASH-MEN) represents the compilation of about 450 new emission line sources identified as PN candidates but now removed as contaminants mostly prior to publication of MASH-I (Parker et al. 2006) and MASH-II (Miszalski et al. 2008). This was achieved by careful application of spectral and multi-wavelength image diagnostic criteria as described in Frew \& Parker (2010).

\section{Methodology}

Several kinds of astrophysical objects mimic planetary nebulae and pre-MASH PN catalogs still contain significant numbers of contaminants that are being detected and 
eliminated via our newly developed methodology. To classify MEN objects into categories, we first collect as much information as possible, i.e. multi-wavelength images and spectra (from surveys as SHS, SSS, DSS, IPHAS, 2MASS, MSX, MIPS, GLIMPSE, and WISE). The $\mathrm{H} \alpha / \mathrm{SR}$ quotient image is effective for revealling the extent of any emission to emphasize the MEN objects. It is also useful to create RGB images which can reveal a reflection component from the B-band. When all these data are available, we are able to classify objects into categories thanks to their morphological and spectroscopic criteria where the overall body of evidence is used in combination with a decision tree we created. Other tools such as diagnostic plots using nebular emission-line ratios are also used to distinguish PNe, Herbig-Haro objects, SNRs, and H II regions. Photometric data are useful to identify symbiotic stars or close-binary CSPN. Other properties used for the identification of the MEN objects are detailed in Frew \& Parker (2010).

\section{Examples and results}
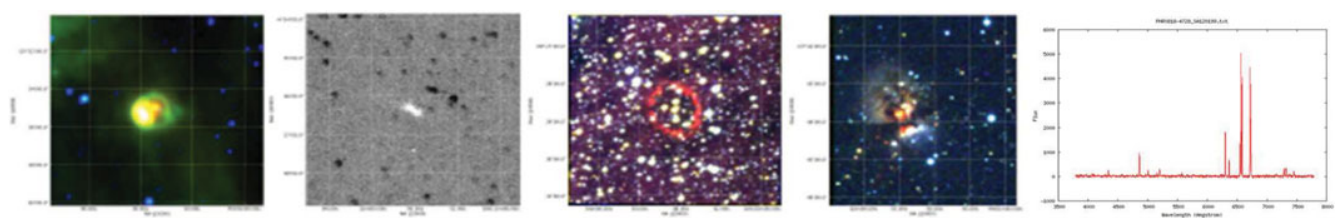

Figure 1. From left to right: PHR0703-1035: HII region (WISE image); PHR1811-0436: symbiotic star with outflows (quotient image); PHR1633-4928: Pop.I WR star with ejecta (SHS image); PHR0901-4743: stars forming region (2MASS image); PHR0818-4728: SNR (spectrum).

All 450 MEN sources (see examples in figure 1) have been classified into about a dozen different object categories. Unsurprisingly, HII regions (39\% of MEN objects) and symbiotic stars $(22 \%)$ are most often confused with PNe. The MEN catalog contains also $8 \%$ SNRs, $5 \%$ flaws, $4 \%$ galaxies, $4 \%$ reflection nebulae, $3 \%$ YSOs and Herbig-Haro objects, $3 \%$ cataclysmic variables, $2 \%$ clusters, $2 \%$ B $[\mathrm{e}]$ stars, $1 \%$ Wolf-Rayet ejecta, and under $1 \%$ LBVs. Finally, as better data became available, several bona-fide PN previously removed from MASH can now be re-integrated as a result of this project uncovering their true PN nature (6\% of the MEN objects).

\section{Conclusion}

The MASH-MEN Project was instigated first to house the many interesting but nonPN mimics uncovered during the MASH survey and subsequently to classify the mimics according to type into a new catalog of miscellaneous emission nebulae (MEN). Application of our careful diagnostic processes have resulted in the MASH PN catalogs being of high purity. Some of these contaminants are very interesting in their own right and deserve follow-up and further study. We are now applying the same processes to other extant PN catalogs to remove their many mimics.

\section{References}

Parker, Q. A., et al. 2005, MNRAS, 362,689

Parker, Q. A., et al. 2006, MNRAS, 373, 79

Miszalski, B., Parker, Q. A., Acker, A., Birkby, J., Frew, D. J., \& Kovacevic, A. 2008, MNRAS, 384,525

Frew, D. J. \& Parker, Q. A. 2010, PASA, 27, 129 